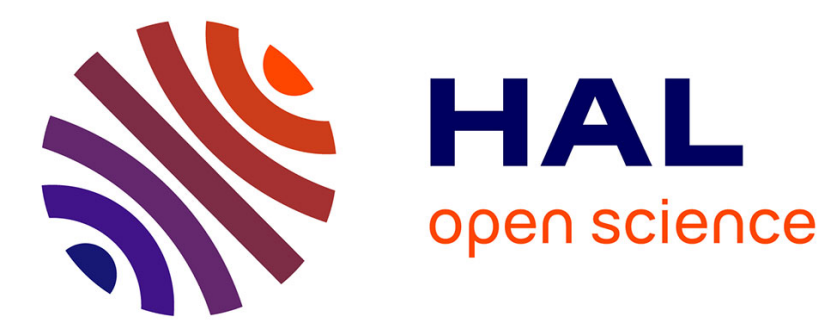

\title{
Viability of Implementing Smart Mobility Tool in the Case of Tunis City
}

Ezzeddine Fatnassi, Olfa Chebbi, Jouhaina Chaouachi

\section{To cite this version:}

Ezzeddine Fatnassi, Olfa Chebbi, Jouhaina Chaouachi. Viability of Implementing Smart Mobility Tool in the Case of Tunis City. 14th Computer Information Systems and Industrial Management (CISIM), Sep 2015, Warsaw, Poland. pp.339-350, 10.1007/978-3-319-24369-6_28 . hal-01444477

\section{HAL Id: hal-01444477 \\ https://hal.inria.fr/hal-01444477}

Submitted on 24 Jan 2017

HAL is a multi-disciplinary open access archive for the deposit and dissemination of scientific research documents, whether they are published or not. The documents may come from teaching and research institutions in France or abroad, or from public or private research centers.
L'archive ouverte pluridisciplinaire HAL, est destinée au dépôt et à la diffusion de documents scientifiques de niveau recherche, publiés ou non, émanant des établissements d'enseignement et de recherche français ou étrangers, des laboratoires publics ou privés.

\section{(c)(1)}

Distributed under a Creative Commons Attribution| 4.0 International License 


\title{
Viability of Implementing Smart Mobility Tool In the case of Tunis City
}

\author{
Ezzeddine Fatnassi ${ }^{1}$ Olfa Chebbi $^{1}$ and Jouhaina Chaouachi ${ }^{2}$ \\ ${ }^{1}$ Institut Supérieur de Gestion de Tunis \\ Université de Tunis \\ 41, Rue de la Liberté - Bouchoucha - 2000 Bardo, Tunisie \\ 2 Institut des Hautes Etudes Commerciales de Carthage \\ Université de Carthage \\ IHEC Carthage Présidence-2016 Tunis, Tunisie
}

\begin{abstract}
Nowadays, different changes from the economical, societal and environmental contexts are happen in cities. In fact, cities are generally the best place to endorse and enhance various experience in order to improve the quality of life of its citizens. In this context, the new vision of Smart Mobility fill into this context. The concept of Smart Mobility as a means to enhance the mobility experience of citizen has been gaining increasing importance in the agendas of cities stakeholder. It represents the best balance the economic, environmental and societal aspect of current transportation tools. The implementation of the smart mobility concept in the case of Tunis city is the subject matter of the paper. In fact, we focus on considering the Personal Rapid Transit system as an effective and efficient tool to bring smart mobility experience to Tunis city. This paper also presents and study the viability of implementing PRT in our specific context. An extensive simulation and economic feasibility study is conducted to validate our proposal. Computational results prove the different advantages of our proposal in the studied context.
\end{abstract}

Keywords: smart city, smart mobility, Personal Rapid Transit, Simulation

\section{Introduction}

It have been observed recently a tremendous increase of urbanization rate. In fact, more and more people tend to move to cities seeking for a better quality of life. The urban population is estimated to represent $53 \%$ of the whole world population. It is expected that 4.5 billion of peoples will be living in cities in $2025^{3}$.

Many cities essentially in Asia and Latin America are expected to have more than 10 million inhabitants and $70 \%$ of the world population are expected to be living in urban areas by 2050.Pressure generated by this high urbanization

\footnotetext{
${ }^{3}$ Source: UN World Urbanization Prospects, World Business Council for Sustainable Development
} 
rate has a main impact on the mobility of peoples and goods in cities. As an example we could note that commuting delay is expected to double to over 100 hours per year per person in 2050. Consequently, it becomes necessary for cities stakeholders to organize and manage urban space and mobility tools to prevent all the negative impact of urbanization on cities.

Cities are generally considered as the right place to develop new initiatives and ideas. In fact, they can contribute to develop and build new innovative ecosystem which could develop new perspectives and opportunities. In this respect, the concept of smart city appears very promising. Smart city concept englobe mainly six different elements (see Figure 1). Among, these elements one could focus on smart mobility and smart environment concepts. These two essential elements of smart cities are closely related to urban mobility. They tends to promote the use of smart public transportation tools that have the least impact on environment.

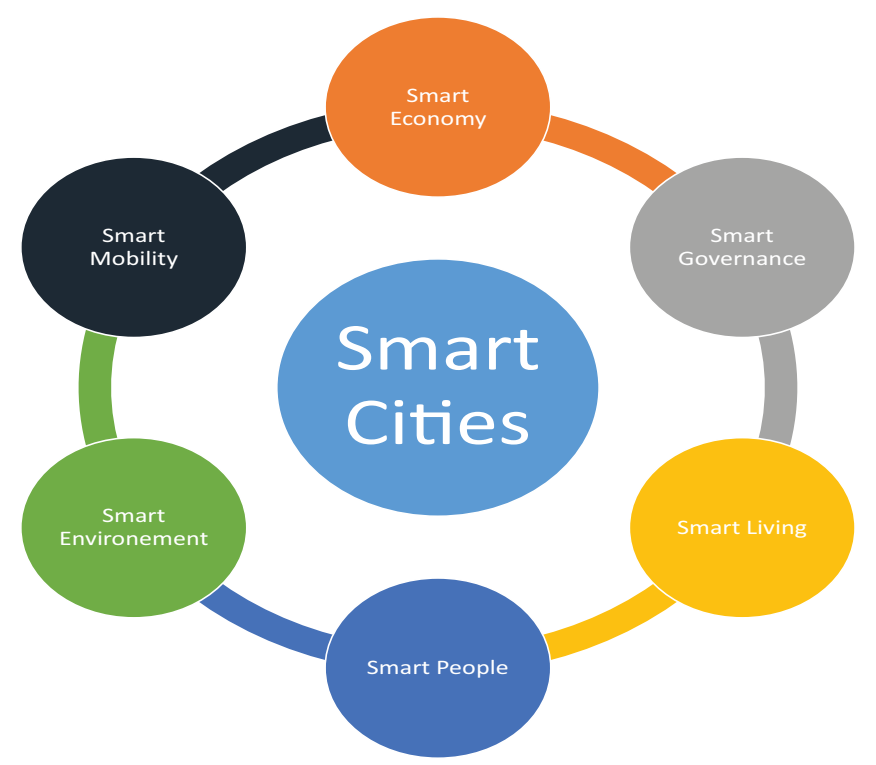

Fig. 1: Smart City Concepts

The recent experience related to smart mobility and smart environment concepts seem to shows that single and individual initiative of a single operating transportation mode to reduce energy consumption and CO2 emissions are note very effective approach is rather necessary to implement the concepts of smart mobility and smart environment. Generally, there is two ways to deal with urban sustainability issue: i) enhance the performance and quality of the already existent transportation tools. ii) Invest and build a completely new sophisticated smart transportation tool. 
In our work, we focused on the second alternative. In fact, the paper is concerned by implementing the concept of smart mobility and smart environment in the case of Tunis city. We aim in our project to introduce a new intelligent transportation tool called Personal Rapid Transit (PRT) and Freight Rapid Transit in Tunis city. The different theoretical aspects related to PRT and FRT are subjects to a close identification in this paper.

The subject of this work is the feasibility of implementing PRT system in the specific context of Tunis city. For that purpose, we develop a new proposal for implementing PRT as an integrated part of a city. We also validate our proposal through simulation and economic feasibility.

In this work and based on the ideas of Smart city and mobility concepts, we propose a combined and integrated PRT/FRT system to be implemented and developed in the case of Tunis city. Next, a formalization of the treated problem is proposed. Then, we 'll focus on an strategic context of the PRT/FRT system to study the economic feasibility of our proposal through the tools of optimization and simulation. Finally, we conclude our paper with general conclusions and insights about our work and present our future research directions.

\section{$2 \quad$ Smart city and mobility concepts}

Smart city is concerned as a relatively new concepts. Smart city is also a highly context dependent. In the literature, there is no clear definition of what smart city is. In fact, in the literature the frontier between smart city concepts and other similar concepts such as intelligent city or creative city is not well defined. That is why many individuals claim that specific city is being smart without following specific directive and standards. However, one could consider that the smart city concept is the natural evolution of cities from a cities as a simple scientific center to a compact enhanced form in which cities use information and communication technologies to increase the efficiency of its different components such as infrastructure, mobility tools, etc [1].

Therefore, one could consider smart city as a conceptual urban development model which is mainly based on the human collective knowledge and technological advance in order to enhance the prosperity and the development of urban centers. That is why the concept of smart city should combine different elements such as the concept of knowledge based cities [2]. Literature about smart cities provides the six most common indicators of smart cities [3]:

- Smart economy: cities should offer a highly attractive economic environment. This should include highly innovative and productive climate as well as a flexible labor market.

- Smart people: Smart people are considered as the results of social and ethnic diversity, creativity, tolerance and so on [2]. To raise the global qualifications as well as a social capital cities could offers online courses or workshop [4].

- Smart governance: Specific city system management are required to reach development in this areas. Services such as e-government and social media 
combined with a transparent decisions making process could lead to smart governance city.

- Smart living: This notion involves the improvement of the quality of live in term of service, environment and social infrastructure. Smart living includes also e-health, social services and specific developed public safety tools. This latter include specific emergency service and surveillance system network [5].

- Smart environment: an efficient smart city should optimize energy consumption as well as reduce the carbon emissions. This could be done through the use of renewable energy sources as well as the implementation of a specific resources management strategies based on sustainability principles.

- Smart mobility: Through specific planning practices, cities could effectively reach the objective of smart mobility. Urban planning strategies would necessarily imply to turn the focus from individuals to collective smart tool of transportation via the use of enhanced information shared and communication technologies.

Focusing on the latter principle, one could note that the idea of smart mobility is crucial for the development of smart cities. Development of smart mobility system means that a city is accessible for its different inhabitant through a safe, robust, eco-friendly and smart transportation tools. The emergence of smart mobility tool would necessarily imply to reach sustainable mobility.

Therefore, developing smart mobility concepts is necessary for any smart city. In the rest of this paper, we present the PRT as an enhanced sophisticated tool to reach the objective of smart mobility.

\section{Personal and Freight Rapid Transit}

The Personal Rapid Transit (PRT) provides a new and innovative system of transportation. PRT offers an ondemand direct transit from origin to destination stations. PRT is oriented to serve passengers in urban areas and is designed to work and act as any traditional taxi system. Therefore, the PRT is highly attractive for its users in comparison with traditional mass transit like bus or subway systems. The Personal Rapid Transit use small electric driverless vehicles (also called pod cars). PRT vehicles run on dedicated guide way in order to provide direct nonstop trips between origin and destination stations. This is made possible by the use of the offline station that is placed off the main line. This specific feature of PRT avoid for pod cars to stop at unnecessary stations. Guideways on such a system can be at grade, on tunnel or elevated and are segregated from other urban traffic and pedestrian. PRT systems contrary to other traditional tools run on a network of guideway rather than a group of simple lines. The network of guideway and the offline stations have the main advantage of being able to be integrated with existent building which reduce the visual intrusion and the time needed to get access to such a system. This is resulted from the small size of the infrastructure as the PRT system use small vehicles. Vehicles in PRT are made to take from 1 to 6 passengers. Therefore, the service quality of PRT is more similar to taxi rather than a bus. 


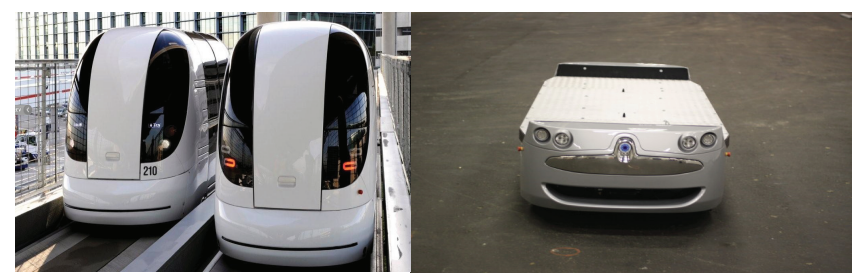

Fig. 2: Example Of PRT ${ }^{4}$ and FRT Vehicle ${ }^{5}$

On the other hand, Freight Rapid Transit is automated people movers that share the same infrastructure and characteristics of a PRT system. However, it is made and designed to transport goods and waste. FRT vehicles could handle up to $1500 \mathrm{Kg}$ and could be adapted to handle different waste freight.

The combination of both PRT and FRT on the same network of guideways presents an interesting alternative for urban zone to mix the flow of passengers and goods on the same shared system. These two intelligent systems combined with all the new technologies that exist nowadays could represent a valid replacement for private vehicles and trucks. This is achieved mainly through different feature and specific characteristics of PRT and FRT:

- They both use electric energy which make them environmentally friendly and energy efficient.

- As the vehicles are driverless, it requires far less human power than other traditional mass transit system.

- As the transit operations are generally made on demand, PRT/FRT offer a better service quality than other transportation systems.

- PRT/FRT are faster than any other transit tools.

\section{Formalization of the Treated Problem}

Implementing PRT system in an urban context require to solve various problems and issue related to that specific urban context. However, these issues could be formalized into a PRT' guideway network design problem. Next, we present the formal problem definition of PRT' guideway network design problem[6].

Generally, a PRT/FRT system contains a guideways network (GN) and a set of PRT and FRT vehicles moving on the network. The GN could be modeled as a partition of a complete directed graph $G=S, \gamma$ where $S=1,2, \ldots, n$ represents the set of $n$ stations and $\gamma=(i, j) \mid i, j \in S, i \neq j$ represents the set of $n(n-1)$ of possible directed links between each couple of stations in $\gamma$. The length of each possible link $(i, j)$ is denoted by $d_{i j}$. The number of vehicles needed to travel from a location $i$ to a location $j$ is denoted by $t_{i j}$.

\footnotetext{
${ }^{5}$ source:http://www.ultraprt.net/multiVehicle.htm

${ }^{5}$ source:http://www.2getthere.eu/?pageid $=912$ /
} 
The input of the GN are the station locations, the set of $d_{i j}$ and $t_{i j}$ where $i$ and $j \in S$ and $i \neq j$. For a GN, two main problems exists: the GN design and the station design problems. The objective of the GN design is to find the least cost possible for building a PRT network while respecting connectivity constraints related to PRT. Connectivity constraints include the possibility for each vehicle to move from any station to any other station. On the other hand, the station design problem is to find the least cost option possible to design a PRT station subject to traffic constraint.

The formal GN design problem definition could be defined as follow:

- Given : $G, S, \gamma, d_{i j}$ and $t_{i j}$.

- Minimize : $f_{c}(x), f_{t}(x)$.

- Subject to connectivity constraints.

Where $f_{c}(x)$ represents the construction costs and $f_{t}(x)$ represents the traffic congestion over the PRT network.

We should note that $f_{c}(x)$ could be defined as follow :

$f_{c}(x)=\Sigma d_{i j} x_{i j}$, where $x_{i j}$ is a binary variable equals to 1 if the link between $i$ and $j$ is selected in the final solution.

On the other hand, $f_{t}(x)$ is a function that aims to minimize the number of vehicles passing a point in the GN in a unit of time. This objective aims to ensure a fluid traffic in the GN.

To evaluate a designed GN, an empty vehicle problem (EVP) could be used. The EVP could be formulated as follow[7].

Given a PRT Network with $(S)$ stations, one depot $D$ and enough links to make possible the move between each couple of stations. This problem deals with empty vehicles movement over a designed GN in a static deterministic context. Therefore, we suppose to have a predefined list of trips $(\Theta)$ of size $\nu$. Each trip $i$ is defined by the following: i)Depart time $D t_{i}$, ii) Depart station $D s_{i}$, iii) Arrival time $A t_{i}$, iv) Arrival Station $A s_{i}$.

We suppose to have an unlimited number of vehicles initially located at the depot. Each vehicle have a Battery capacity $B$. The problem is defined on a Graph $\chi=V, E$ where $V$ is the set of nodes and $E$ the set of arcs.

The set $V$ contains $\nu$ nodes that represent the $\nu$ trips plus two dummy nodes $\varsigma$ and $\tau$ that represent the depot. We define also $V^{*}=V /\{\varsigma, \tau\}$. The set of edges $E$ defined on the following rules:

- for each node $i, j \in V^{*}$ with $j>i$ and, the arc $(i, j)$ exists if $A t_{i}+$ $\operatorname{cost}\left(A s_{i}, D s_{j}\right)<=D t_{j}$ the cost of this arc will be the cost of moving from the arrival station of trip $i$ to the arrival station of station $j$.

- for each node $i \in V^{*}$ the arc $(\varsigma, i)$ exists and have as a cost the energy used to go from the depot to the arrival of station of trip $i$.

- for each node $i \in V^{*}$ the arc $(i, \tau)$ exists and have as a cost the energy of moving from the arrival station of station $i$ to the depot.

The objective of solving our problem is to evaluate a designed GN while treated a specific scenario determined by the list of trips $\Theta$. Therefore, finding 
a set of least cost vehicles roads that satisfy the different trips in $\Theta$ could be used as a valid benchmark to evaluate a designed GN. Such a scenario could be obtained using historical data of traveling passengers in a predefined urban areas.

\section{$5 \quad$ Proposal Of Implementing PRT}

This work aims to evaluate the feasibility of implementing PRT as a smart mobility tool in Tunis city. Tunis city is suffering like many a high urbanization rate phenomena with all its negative impact on people mobility. It is a strong intention from the city authorities to launch different measures that could relieve the already existent transportation tools. Thus making the urban center more attractive to visitors, tourists and residents.

\subsection{Local characterization}

Tunis city is the capital of Tunisia located at the north east of the country. It presents a total area of 212,63 and it has a total population of 728,453 which corresponds to a density of 3426 inhabitant $/ \mathrm{km}^{2}$. The total Tunis metropolis area have a total number of 2721227 habitant. Considering only the areas regarded as the Tunis urban center (see Figure 3), it has been reported in [8] that it has a total of $10 \%$ of the whole population of the metropolitan area of Tunis with $30 \%$ of the total jobs [8]. It will remain for topographical reasons a center place of crossing for the main urban and goods flows within the metropolitan area. The main and only available public transportation tool to move in Tunis urban center is the light rail transit (LRT).

LRT (also called Metro Leger in French but it is more like a tramway than a metro) was first implemented in 1985 by operating the south line after it was decided in 1980. The implementation of the LRT network has suffered many uncertainties due to the various policies makers that disagreed its implementation. But finally, the whole project was implemented while constructing 6 different lines during the period 1988-2008. The LRT current network have a total length of $45 \mathrm{Km}$ of double track, 6 lines, two main transfer stations (Barcelone and La République which are located at the urban center of Tunis). The total LRT ridership was around 35000 daily passengers[9]. The LRT in Tunis city suffers from many limits. Among them one could consider its limited capacity. The loading rate of the LRT cars is about $100 \%$ and for various lines, it excess $100 \%$ especially in the morning peak.

An estimation of the real modal share give the majority of the urban trips to private cars with $60 \%$. This fact is suggested by the high ridership rate of $100 \%$ of the LRT line. This results of the difficulty for the private cars drivers to leave their cars for a public transportation tool.

Therefore, we could state that the context of Tunis urban center is characterized by a high use of private cars, a high rate of congestion with a limited option for implementing new mass transit solutions. 
Following all these conditions, it becomes of a high interest to impose the quality and the capacity of public transportation tools in Tunis urban center. In the next, we propose an illustrative example and study into the urban center of Tunis with LRT to improve the quality of public transportation tools.

\subsection{Integrated PRT solution for the urban center of Tunis}

After the different conclusion exposed in the last section, it appears necessarily to launch a new public transportation plan to overcome different issues. As underlined above, the systematic view of PRT is crucial for its integration in the current transportation practices. To append this systematic view on PRT, a mutualisation of the available resources (the LRT stations, available urban areas in the urban center, etc.) for a specific joint usage. It is also of a high interest to plan for a shared usage between PRT and LRT as it offers various options for city planner and managers. In fact, the opportunity to plan for a shared travels is among the recent trends in managing transportation systems.

We also foreseen a specific interest to design beforehand an integrated PRT network in order to allow persons to benefit from a highly sophisticated intelligent transportation tool fully connected to the LRT network. We should note also that the LRT network in the Tunis urban center is not dense and do not covers many essential commercial areas in the urban center and the last mile delivery has to be developed and implemented.

Therefore and to better explain our PRT implementation proposal, an example is depicted in Figure 3. In this illustration, we propose to implement a PRT network that follow the line of the LRT network. Our proposition aims to use elevated guideways in a specific way as shown in Figure 3. We also aim to use the already existent main station as intermodal facilities in order to allow users easily for LRT to PRT. Our option don't interfere with other urban projects as the areas that will be used for PRT is already in use by the LRT. Also, the small size of PRT guideways and station allows easy integration with its urban context. Our proposal encompass 8 stations and $45 \mathrm{~km}$ of one way guideways. is very beneficial for the users of LRT as they could easily be transferred from LRT vehicles to PRT vehicles in order to reach easily their destination.

This scheme is designed to solve the recurrent problem of congestion and capacity for LRT in urban center of Tunis. This option aims also to offer a smart mobility tool for the resident of Tunis and a new image for the city center which will be very beneficial as tourists could be attracted to the city center. It is clear from this proposal of an integrated LRT/PRT in the center of Tunis must go through three levels of decisions: It is clear that the implementation of an integrated PRT/FRT network, must go through a three levels decision-making process in order to determine:

- How to design the network and its transit capabilities (deployment, vehicles, stations).

- How to plan and schedule for the number of PRT/FRT and their capacities.

- How to optimize the travels in terms of energy use and transit time. 


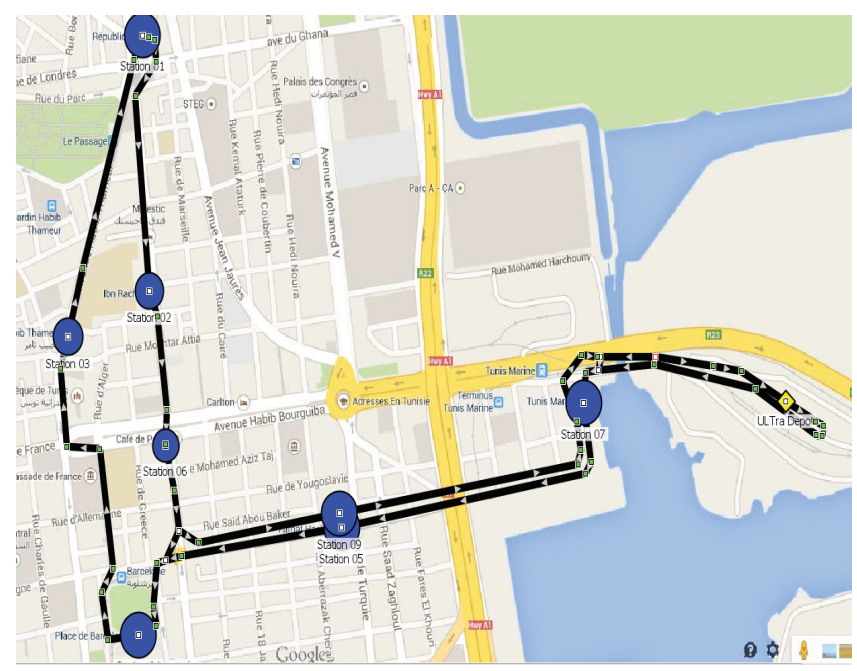

Fig. 3: Proposal Of Implementing PRT in Tunis City

The rest of this paper will focus on the feasibility of such a proposal and its related economic benefits.

\section{Simulation and Economic feasibility}

In order to test the feasibility of our proposal it was necessary to perform simulation study. In the literature, there are several simulation software for PRT. We used in this work, the ATS/mobile simulation software. In fact, this software enable its users to import specific maps and draw and test PRT feasibility. Demand scenarios was calibrated according to real condition on the LRT network. We run our simulation for 2 hours. We performed 10 different tests in order to

eliminate any variability issues ${ }^{6}$. ${ }^{6}$ the simulation output shows that the PRT would offer an average waiting time of 19.354 seconds for its users( see Figure 4). The PRT in just 2 hours was able to perform and satisfy on average 1986.9 trips. This with a projection on a typical whole working day (7AM to $19 \mathrm{AM}$ ) makes the PRT able to satisfy up to 20,000 trips per day. This feature allows it to attract many users as it offers a really low waiting time.

\subsection{Economic feasibility}

In this section, we present a preliminaries economic feasibility of implementing PRT in the PRT urban center. The overall proposed system costs are the sum

\footnotetext{
${ }^{6}$ The experiments were performed on a computer with a $3.2 \mathrm{GHZ}$ CPU and 8 GB of RAM.
} 


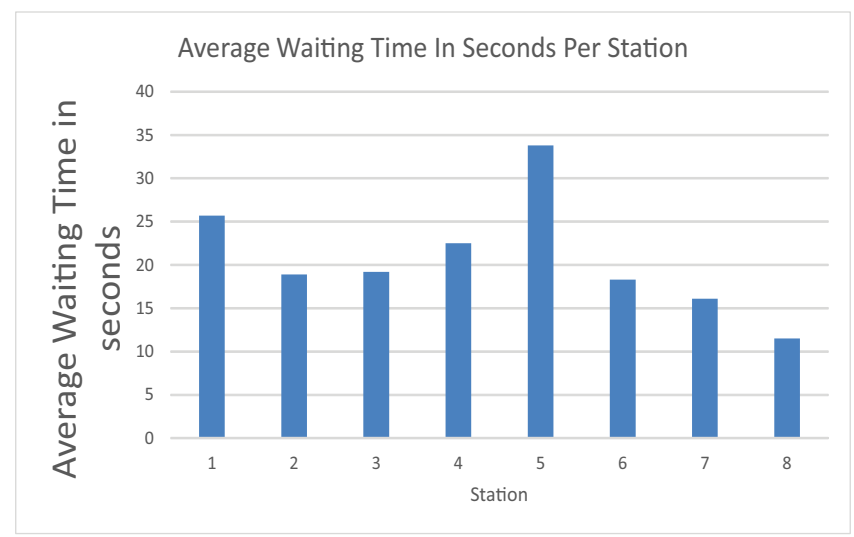

Fig. 4: Results Of The Simulation

of the annual capital costs, maintenance costs and day to day operations costs. In order to estimate the PRT fare which exceeds the total system costs, we performed a calculation based on the general costs and on the simulation results to estimate the operational costs.

Computing system costs The system costs involves mainly all the different investments that need to be made in order to implement the proposed PRT system. The capital costs englobe the infrastructure costs (station, tracks, etc.), the cost for the different communication systems as well as the cost for the purchasing the PRT vehicles. The costs are then distributed over the period of time until that all the assets need replacement. Following this methodology allow us to obtain specific annual capital costs. We also supposed to use debt as a financing resources for the initial investment. Following these assumptions, interest rate should be considered.

Operating costs on the other hand are represented by the energy costs to run the PRT vehicles and the salaries for the workers on the PRT system. Finally, maintenance costs occurs for repairing, cleaning the vehicles and the different infrastructure. After obtaining all these values, the annual systems costs is computed as shown in table 1 .

Computing the PRT fare After obtaining the costs for a PRT system, we need to estimate the PRT fare per Km which could cover the total system costs. For that purpose, we used the different simulation output in order to estimate the PRT fare which corresponds to the simulated PRT trips 'demand per day as well as the average trips length in $\mathrm{Km}$.

From the obtained results, we should note that a minimum of $0.19 \$$ per $\mathrm{km}$ is required to cover the total system costs. We should note that the system covers zones that are not mainly covered by other transportation tools except for LRT. We should note that buses and LRT in Tunis costs, LRT costs $0.16 \$$ and taxi 
costs $0.59 \$$ per $\mathrm{km}$. Those transportation tools are less convenient than PRT. Hence, a fare between $0.2 \$$ and $0.3 \$$ per $\mathrm{Km}$ could be well acceptable by the population.

\section{Conclusions}

During this study, it was possible to realize the importance of implementing smart mobility tool in dense urban area in a developing country. We presented PRT as a promising transportation tool to enhance the efficiency of the already existent transportation options. In that way, we proposed a specific PRT proposal which would integrate PRT to the urban context in Tunis city. We then performed a simulation and feasibility study in order to test the feasibility of our proposal. Our preliminaries results are very insightful on how our propositions could be improved. In our future work, the development of an enhanced simulation study could be done. Also, we would like to extend on managerial insight on how PRT could be successfully implemented and integrated with other transportation tools.

\section{References}

1. Dewalska-Opitek, A.: Smart city concept-the citizens perspective. In: TelematicsSupport for Transport. Springer (2014) 331-340

2. Letaifa, S.B.: How to strategize smart cities: Revealing the smart model. Journal of Business Research (2015)

3. Allwinkle, S., Cruickshank, P.: Creating smart-er cities: An overview. Journal of urban technology 18(2) (2011) 1-16

4. Steinert, K., Marom, R., RICHARD, P., Veiga, G., WITTERS, L.: Making cities smart and sustainable. The Global Innovation Index 2011 (2011) 87

5. Hielkema, H., Hongisto, P.: Developing the helsinki smart city: the role of competitions for open data applications. Journal of the Knowledge Economy 4(2) (2013) 190-204

6. Won, J.M., Lee, K.M., Lee, J.S., Karray, F.: Guideway network design of personal rapid transit system: A multiobjective genetic algorithm approach. 2006 IEEE Congress on Evolutionary Computation, Vols 1-6 (2006)

7. Mrad, M., Hidri, L.: Optimal consumed electric energy while sequencing vehicle trips in a personal rapid transit transportation system. Computers \& Industrial Engineering 79 (2015) 1-9

8. Baltagi, A.: Projet d un reseau integre de transports collectifs dans la ville de tunis. In: CODATU XI: world congress: Towards more attractive urban transportation. (2004)

9. Godard, X.: Some lessons from the lrt in tunis and the transferability of experience. Transportation Research Part A: Policy and Practice 41(10) (2007) 891-898 
Table 1: PRT Cost

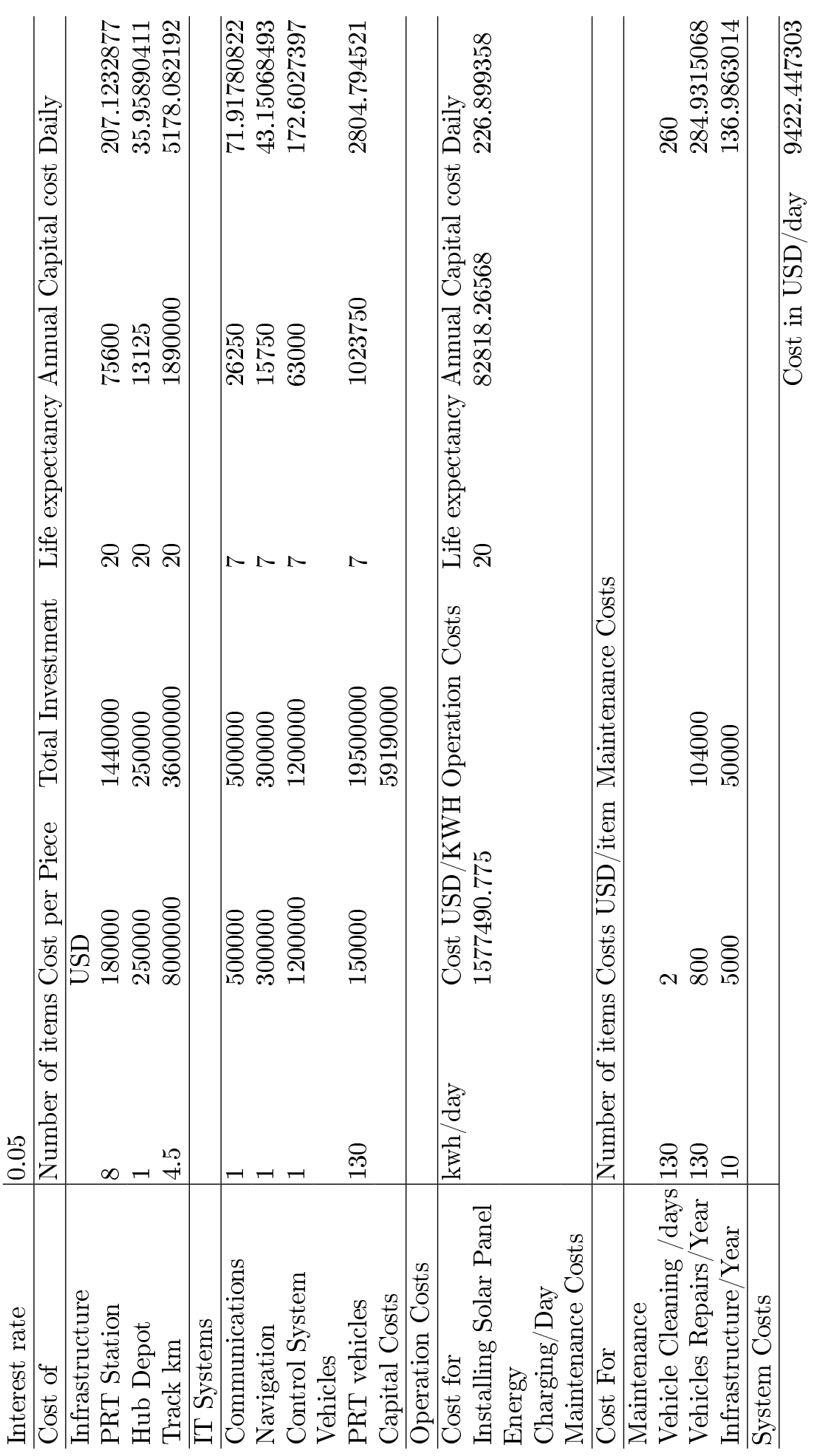

